\title{
THOUGHTS ON COMMON PRACTICAL PROCEDURES
}

\author{
Major A. J. SPICER, M.B., B.S., M.R.C.P., D.C.H., R.A.M.C. \\ British Military Hospital, Iserlohn
}

IT is felt that the techniques described in this paper have advantages over those more usually employed. Whilst there is nothing new about some of them, they deserve wider publicity and use. Safety and comfort for the patient and efficiency and convenience for the operator are factors to be considered for every technique.

\section{Pleural aspiration}

The standard apparatus for aspirating fluid from the pleural cavity is a chest aspiration needle, $50 \mathrm{cc}$ glass syringe and a two-way metal tap. The needle is too long and is difficult to maintain at the correct depth of insertion. The patient must remain still throughout the procedure. The syringe and tap tend to stick and the apparatus is uncomfortable to use. As a result of lung expansion, or movement by the patient or the operator, there is a risk of damaging the lung with the needle and producing a haemothorax, pneumothorax or air embolism.

The combination of a $20 \mathrm{cc}$ plastic syringe, plastic two-way tap (Figure 1.B), a Braun size 3 intravenous trocar-needle and cannula (A) and a length of plastic tubing (D) makes an ideal chest aspiration apparatus. It is light and comfortable to use and neither the syringe nor the two-way tap are prone to stick. The Braun plastic cannula is flexible, atraumatic, of adequate diameter and ideal length. All components are sterile packed and disposable.

The site of aspiration is determined by careful clinical examination and posteroanterior and lateral X-rays. Unless the fluid is loculated, the site is usually in the ninth intercostal space in the same longitudinal line as the inferior angle of the scapula. The patient sits forward with arms resting on a hospital bed table. The chest wall is infiltrated with a local anaesthetic. With the syringe fitted to the trocar-needle and cannula and exerting continual suction, the needle is advanced through the intercostal space until fluid is aspirated. The trocar-needle is then withdrawn and the syringe fitted to the cannula. The cannula is of such a length that the hand supporting the syringe can be steadied against the chest wall. This minimises movement of the cannula inside the pleural cavity and it is particularly important when it is necessary to change the patient's position in order to complete the aspiration.

\section{Insertion of an intercostal catheter for pneumothorax}

The widely accepted treatment for a moderate to large sized pneumothorax is to remove the air by inserting an intercostal catheter and attaching it to an under-water seal or valve. A wide variety of catheters and tubes designed for other purposes are used and are inserted with the aid of a trocar and cannula. The large diameter of the cannula and the force required to insert it makes it an unpleasant procedure for the patient, is liable to damage intercostal vessels and nerves and leaves a scar.

The Argyle thoracic catheter (Figure 1, E) is specifically designed for closed drainage of the pleural space. It is a transparent plastic tube with an aluminium trocar. It has terminal and sub terminal holes, a radio-opaque marker and the proximal end is funnel 


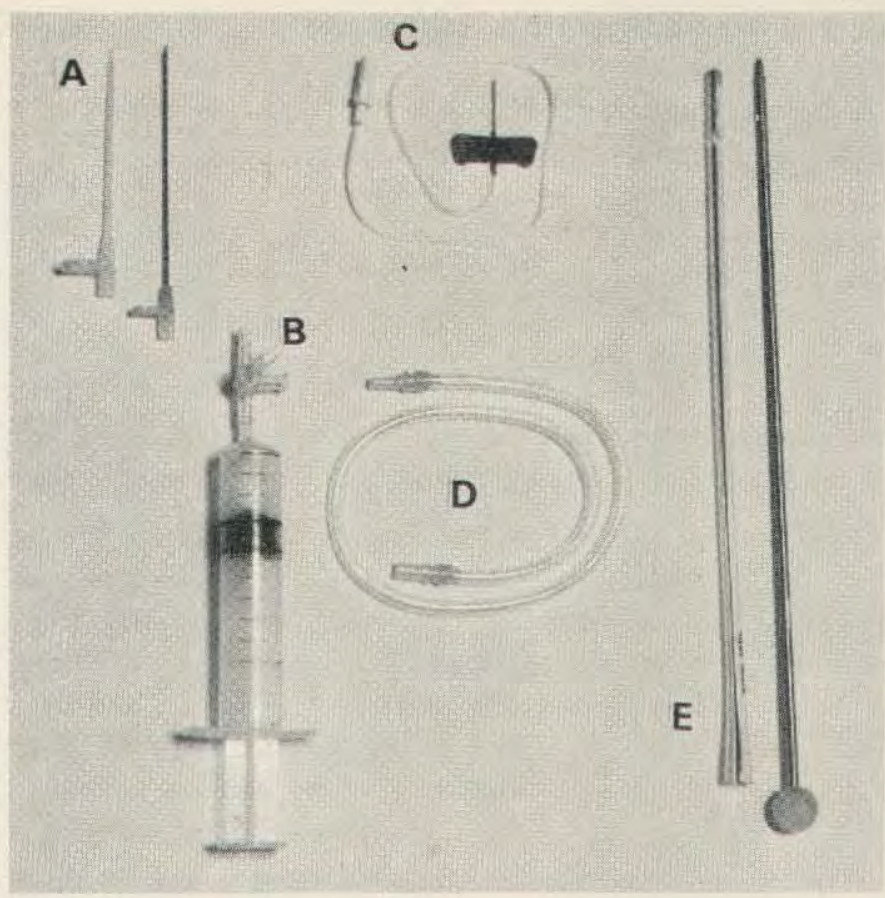

Fig. 1. Thoughts on Common Practical Procedures.

shaped to accommodate a variety of connectors. The catheter is flexible but is not liable to kink or angulate. It is available in sizes 12 to 28 French gauge.

The usual site of insertion is in the second intercostal space in the mid-clavicular line. After infiltration of the chest wall with a local anaesthetic, the skin is nicked with a scalpel. The trocar and catheter are inserted with moderate pressure and a rotating action, close to the upper border of the third rib to avoid the intercostal vessels and nerves. The trocar is withdrawn and the catheter connected to an under-water seal or valve. The position of the catheter is determined by X-ray and adjusted if necessary. Expansion is usually complete after 24 to 48 hours, unless there is a further leak of air from the lung, or there is atelectasis due to bronchial obstruction by a mucus plug.

\section{Arterial puncture}

Knowledge of blood gas levels is essential for the proper management of respiratory failure. It is an important aid to the diagnosis of pulmonary embolus. There is a fairly good correlation, statistically, between the $\mathrm{P}_{\mathrm{O} 2}$ levels of arterial blood and " arterialised" capillary blood. It does not apply to all patients, however, and capillary blood estimations should not be relied upon. This will be discussed in a subsequent paper.

The radial artery has the advantages of being readily palpable, often visible and crossing a rigid platform of bone. A small volume of heparin, 25.000 units per $\mathrm{cc}$, is drawn into a $10 \mathrm{cc}$ plastic syringe and ejected through the $25 \mathrm{~g} \mathrm{x} 15 \mathrm{~mm}$ intradermal needle which is used for arterial puncture. The residual heparin prevents the aspirated 
blood from clotting. With the wrist held in dorsiflexion, the needle is inserted at a right angle to the skin and the artery transfixed against the underlying bone. Whilst continual suction is exerted with the syringe the needle is slowly withdrawn until the lumen of the artery is entered and blood aspirated. On completion of the procedure the syringe is sealed by inserting the needle into a rubber cork. Local analgesia is not required, but infiltration of local anaesthetic around the artery minimises the possibility of spasm. It has the disadvantage of making the artery more difficult to define. Arterial puncture is a safe and simple procedure.

\section{Venous blood sampling in infants}

Difficulty is often experienced in obtaining adequate blood samples from infants. The small superficial veins are difficult to penetrate and readily collapse when aspiration of blood is attempted.

The external jugular is the largest visible vein in infants and is usually the best for blood sampling. The infant should be securely swaddled in a sheet and held on its side on a couch, with the body supported between an assistant's forearms and the head dependant and firmly held in slight lateral flexion. The operator gently compresses the vein with a finger at the root of the neck and the needle is inserted along the line of the vein. Occasionally blood cannot be obtained by this method from overweight babies with short necks. An alternative procedure is to insert a scalp vein needle (Figure 1, C), with the plastic tubing cut to about $1 \mathrm{~cm}$ in length, into a scalp vein and the blood allowed to flow into the bottle. The rate of flow is increased by compressing the vein proximal to the site of insertion. Haematoma formation is minimised by comforting the infant in an upright position and lightly pressing on the venepuncture site when sampling is completed.

\section{Administration of intravenous therapy}

It is sometimes difficult in the obese and in shocked and dehydrated patients to find a suitable vein in order to administer intravenous fluids. Long term administration of intravenous therapy, as in bacterial endocarditis, presents a problem as medium sized veins become thrombosed and inflamed. During slow bolus injections to the young and restless the needle is often displaced from the vein, which produces pain and tissue necrosis with some drugs.

An infant scalp vein needle is easily and almost painlessly inserted into the smallest superficial veins. When it is sited in the front or back of the forearm splinting is not necessary and full use of the hand can be allowed. Because small veins are used the site of insertion can be frequently changed, which minimises the problems of administration of long term therapy. Small veins usually remain patent for three to six days. Through an Abbott 21ga "Butterfiy" scalp vein needle inserted in a small vein, one litre of electrolyte can be given in about two hours and a unit of whole blood in two to three hours. In emergency situations it is quicker and simpler to establish two intravenous drips with scalp vein needles than cannulate a collapsed medium sized vein, or perform a cut down. For slow bolus injections the plastic tubing which joins the needle to the syringe minimises the risk of displacing it from the vein. 


\section{Equipment}

Braun intravenous trocar-needle and cannula-Armour Pharmaceutical Company Ltd., Eastbourne, Sussex.

Plastic two-way tap-Rocket \& Co. Ltd., Watford, Herts.

Plastic extension tube-Capon Heaton \& Co., Ltd., Birmingham.

Argyle thoracic catheter-Sherwood Medical Industries, Feltham, Middlesex.

Abbott scalp vein needle-Abbott Laboratories, North Chicago, Illinois 60064.

\section{Preliminary Announcements-Corps Occasions for 1973}

\section{Provisional dates are as follows:-}

R.A.M.C. Annual General Meetings of General and Officers Funds,

R.A.M.C. Association and Corps Mess Fund

27 April

8 June

Army Medical Services Swimming Gala

Conferring of the Freedom of the Borough of Aldershot on the Army Medical Services

27 June

R.A.M.C. Officers' Dinner (7-15 for 8 p.m.)

5 July

R.A.M.C. Officers 'At Home' (4-6 p.m.)

6 July

R.A.M.C./R.A.D.C. Sports

7 July

Army Medical Services Drumhead Service at R.A.M.C. Training Centre and R.A.M.C. Association Annual Lunch

8 July

R.A.M.C. Golfing Society--Spring Meeting-Worplesden Golf Club

4 May

Summer Meeting-North Hants Gold Club

25 June

Autumn Meeting-West Hill Golf Club

16 October

5 October

R.A.M.C. (Q.M. Adm. \& Tech.) Officers' Dinner Club

(For further details see the Army Medical Services Magazine, Winter Number) 